\title{
The Role of the Primate Amygdala in Conditioned Reinforcement
}

\author{
John A. Parkinson,, ${ }^{1}$ Harriet S. Crofts, ${ }^{2}$ Mike McGuigan,, ${ }^{1}$ Davorka L. Tomic,, ${ }^{1}$ Barry J. Everitt, ${ }^{2}$ and \\ Angela C. Roberts ${ }^{1}$
}

\author{
Departments of ${ }^{1}$ Anatomy and ${ }^{2}$ Experimental Psychology, University of Cambridge, Cambridge CB2 3DY, \\ United Kingdom
}

Conditioned reinforcement refers to the capacity of a conditioned stimulus to support instrumental behavior by acquiring affective properties of the primary reinforcer with which it is associated. Conditioned reinforcers maintain behavior over protracted periods of time in the absence of, and potentially in conflict with, primary reinforcers and as such may play a fundamental role in complex social behavior. A relatively large body of evidence supports the view that the amygdala (and in particular the basolateral area) contributes to conditioned reinforcement by maintaining a representation of the affective value of conditioned stimuli. However, a recent study in primates (Malkova et al., 1997), using a second-order visual discrimination task, suggests that the amygdala is not critical for the conditioned reinforcement process.

In the present study, excitotoxic lesions of the amygdala in a new world primate, the common marmoset, resulted in a pro- gressive impairment in responding under a second-order schedule of food reinforcement. In addition, the responding of amygdala-lesioned animals was insensitive to the omission of the conditioned reinforcer, unlike that of control animals, for which responding was markedly reduced. In contrast, lesioned animals were unimpaired when responding on a progression of fixed-ratio schedules of primary reinforcement. These data confirm that the amygdala is critical for the conditioned reinforcement process in primates, and taken together with other recent work in monkeys, these results suggest that the contribution of the amygdala is to provide the affective value of specific reinforcers as accessed by associated conditioned stimuli.

Key words: appetitive conditioning; marmoset; excitotoxic; goal-directed behavior; incentive value; second-order schedule
Conditioned reinforcement is a process by which stimuli in the environment can control and maintain behavior in the absence of primary reinforcers such as food, sex, and warmth. Conditioned reinforcers acquire their motivational properties through direct Pavlovian association with primary reinforcers [i.e., they are Pavlovian conditioned stimuli (CS)] and subsequently act themselves as goals for actions. In the laboratory, conditioned reinforcers such as a light paired with food, sex, or drugs can produce high rates of instrumental responding over protracted periods of time in both monkeys and rats (Goldberg, 1973; Katz, 1979; Everitt et al., 1989; Everitt, 1990; Arroyo et al., 1998). The process of conditioned reinforcement may underlie a great deal of human behavior (Williams, 1994) and may contribute to complex social phenomena, including drug dependence (Altman et al., 1996) and decision-making (Damasio, 1994).

The involvement of the amygdala in conditioned reinforcement has been, until recently, unequivocal. Gross ablation of the amygdala in rhesus monkeys, which also causes nonspecific damage to fibers of passage and to the adjacent rhinal cortex, produced impairments on a task requiring subjects to solve a series of visual discriminations in which the only feedback that the monkey

Received April 27, 2001; revised June 21, 2001; accepted July 13, 2001.

This work was supported by a Medical Research Council (MRC) Career Establishment Grant (A.C.R.) and is a publication within the MRC Cooperative on Brain, Behaviour, and Neuropsychiatry. We thank Prof. A. Dickinson for useful discussions, Dr. R. M. Ridley for supplying the marmosets, C. H. Morrison and R. Underwood for preparation of histological material, J. Bashford for photographic assistance, and I. Bolton and A. Newman for help with preparation of figures.

Correspondence should be addressed to John A. Parkinson, Department of Anatomy, University of Cambridge, Downing Street, Cambridge CB2 3DY, UK. E-mail: jap22@cam.ac.uk.

Copyright (C) 2001 Society for Neuroscience $\quad 0270-6474 / 01 / 217770-11 \$ 15.00 / 0$ received after each response was the presentation of a conditioned positive or negative auditory stimulus. Using this feedback, monkeys earned primary reinforcement only when they made four consecutive responses to the discriminanda associated with the positive conditioned stimulus (Gaffan and Harrison, 1987). Subsequently, more selective neural manipulations of the amygdala in rats, including excitotoxic lesions and central infusions of glutamate receptor antagonists, have identified the basolateral area (comprising the lateral, basal, and accessory basal nuclei) as a critical site involved in instrumental responding with conditioned reinforcement (Cador et al., 1989; Everitt and Robbins, 1992; Burns et al., 1993; Whitelaw et al., 1996; Gewirtz and Davis, 1997; Meil and See, 1997). However, a recent attempt to replicate the original findings of Gaffan and Harrison (1987) in monkeys using selective excitotoxic lesions of the amygdala found that lesioned monkeys were capable of completing the secondorder visual discrimination task without significant impairment (Malkova et al., 1997). Based on the results of a food-devaluation experiment (Malkova et al., 1997), the investigators suggested that the amygdala is either involved only in the acquisition, but not performance, of conditioned reinforcement, or instead is specifically involved in acquiring an association between environmental stimuli and the value of one particular foodstuff compared with another, and not the association between environmental stimuli and food reward as opposed to no reward.

An alternative explanation for the apparent discrepancies is that performance on the procedure used by Malkova et al. (1997) was not sufficiently dependent on conditioned reinforcement processes to be disrupted by amygdala lesions, either because of the extensive pretraining period used or because of the low response 
requirements of the schedule to obtain primary reinforcement. Therefore, the present study set out to assess whether the primate amygdala is involved in conditioned reinforcement, by using a second-order schedule of responding with conditioned reinforcement. In this procedure instrumental responding for primary reinforcement can be maintained over protracted periods of time through the presentation of a conditioned reinforcer (Mackintosh, 1974). Performance on such second-order schedules has been shown previously to be highly sensitive to basolateral amygdala lesions in rats (Everitt et al., 1989; Whitelaw et al., 1996). In the present study, the response requirements were made progressively greater to tax the control over behavior by conditioned reinforcement. Selective, axon-sparing excitotoxic lesions were made after acquisition of conditioning to the tone as in previous studies (Gaffan and Harrison, 1987; Cador et al., 1989; Malkova et al., 1997). The contribution of the CS in maintaining responding was assessed directly by including a critical test session in which the presentation of the conditioned stimulus was omitted during responding (CS-omission tests) (Katz, 1979; Arroyo et al., 1998). To test for any effects of the amygdala lesions on the responding governed by primary reinforcers or on the generalized effects on motivation, all animals were also tested on a series of fixed-ratio (FR) response schedules for primary reinforcement (i.e., responding for primary reinforcement in the absence of any conditioned stimuli).

\section{MATERIALS AND METHODS \\ Subjects}

Twelve common marmosets (Callithrix jacchus), six females and six males, were used in the present study. Mean age at the outset of testing was 24 months. All were housed in pairs. After the daily session of behavioral testing, monkeys were fed $20 \mathrm{gm}$ of MP.E1 primate diet (Special Diet Services, Essex, UK) and two pieces of carrot. This diet was supplemented on the weekends with additional fruit, eggs, bread, marmoset jelly (Special Diet Services), and peanuts. Because the primary reinforcer used in these experiments was a liquid reinforcer, namely banana milkshake, animals were water restricted for $22 \mathrm{hr} / \mathrm{d}$, having access to water only at the end of the testing day. All procedures were conducted in accordance with the project and with personal licenses held by the authors under the UK Animals (Scientific Procedures) Act of 1986 .

\section{Surgery}

All marmosets were anesthetized with a combination of an injection of ketamine sulfate $(0.05 \mathrm{ml}$, i.m.) (Pharmacia and Upjohn, Crawley, UK) followed by an injection of saffan $(0.4 \mathrm{ml}$, i.m.) (Schering-Plough, Welwyn Garden City, UK) and maintained with supplementary doses of 0.3 $\mathrm{ml}$ of saffan for the duration of surgery. Monkeys were held in a stereotaxic frame with specially adapted incisor and zigoma bars and received either an excitotoxic lesion of the amygdala $(n=6)$ or a sham operation $(n=6)$; groups were matched according to their acquisition of presurgical behavioral responding. Because of the inherent individual variability in brain size, infusion coordinates were tailor-made for each animal. This was accomplished using a standardization technique that has been described in detail previously (Dias et al., 1997) and involved measuring the depth of the frontal pole of an individual marmoset's brain to determine whether adjustments to the standard lesion coordinates were necessary.

Injections of excitotoxin were targeted primarily at the lateral and basal nuclei of the marmoset amygdala, the region shown previously in rats to be involved in conditioned reinforcement (Everitt et al., 1989). A solution of 0.09 м quinolinic acid (Sigma-Aldrich, Poole, UK) was infused bilaterally into the amygdala at the following coordinates from the interaural line: anteroposterior (AP), +9.3; lateral (L), \pm 5.6 ; dorsoventral (DV), +4.0 (0.4 $\mu$ l per hemisphere) and AP, +9.3; L, $\pm 5.6 ; \mathrm{DV}$, +5.0 ( $0.4 \mu \mathrm{l}$ per hemisphere). Sham-operated controls underwent the same surgical procedure as lesioned animals but received infusions of sterile phosphate buffer vehicle rather than excitotoxin. For all placements, inf usions were made over $100 \mathrm{sec}$ through a stainless steel cannula
(30 gauge) attached to a $2 \mu$ l precision Hamilton sampling syringe (Precision Sampling Co., Baton Rouge, LA). The cannula then remained in place for $4 \mathrm{~min}$ before being withdrawn slowly.

After surgery, all animals were administered glucose and saline solution $(0.9 \%$ saline, $1 \%$ sucrose; $5 \mathrm{ml}$, i.p.) followed by intramuscular administration of Valium (Roche, Hertfordshire, UK) in the range of $0.05-0.25 \mathrm{ml}$ intermittently over the first $24 \mathrm{hr}$ to suppress any epileptic seizure activity.

\section{Histological analysis}

All monkeys were perfused transcardially with $500 \mathrm{ml}$ of $0.1 \mathrm{M} \mathrm{PBS}, \mathrm{pH}$ 7.4 , followed by $500 \mathrm{ml}$ of $0.4 \%$ paraformaldehyde fixative administered over $\sim 10 \mathrm{~min}$. The entire brain was removed and placed in fixative solution overnight before being transferred to a $30 \%$ sucrose solution for a minimum of $48 \mathrm{hr}$ before sectioning. Sections were cut on a freezing sledge microtome at a thickness of $40 \mu \mathrm{m}$. Every fifth section was mounted on a gelatin-coated glass microscope slide and stained with cresyl fast violet. An additional set of sections was prepared for immunohistochemical staining using the neuronal nuclear protein antibody NeuN (Yakovlev et al., 1997). This provides a selective stain for neuronal cell bodies and allows a precise assessment of neuronal density, and hence cell-sparse lesioned areas.

Both cresyl violet- and NeuN-stained sections were used to identify the lesioned area, which was defined by major neuronal loss often accompanied by marked gliosis. The size and extent of the lesion for each marmoset was then schematized onto drawings of coronal sections through the marmoset brain at the level of the amygdala complex, and a composite diagram was then created illustrating the extent of overlap between lesions (Fig. 1). In addition, for one marmoset that was deemed to have a representative amygdala lesion, the lesion was documented photographically at both high and low magnification using cresyl-stained sections (Fig. 2).

\section{Behavioral methods}

\section{Apparatus}

All testing took place in a specially designed automated test apparatus located within a sound-attenuated chamber. This apparatus has been described in detail previously (Roberts et al., 1992) and in essence allowed animals to make responses on a touch-sensitive visual display unit (VDU). Correct responses were rewarded with banana milkshake, an unconditioned reinforcer, presented through a lick-tube positioned centrally in front of the VDU, and also with an auditory tone, operating as a conditioned reinforcer, presented through two speakers positioned on either side of the VDU. An illustration of the apparatus and an account of the preliminary touchscreen training procedure can be found in Roberts et al. (1992).

All programs were written by the authors in Arachnid Control Language (Cenes Ltd., Cambridgeshire, UK) running on Acorn Risc PCs. Visual stimuli were colored squares $(32 \times 32 \mathrm{~mm})$ presented on either side or in the center of the screen.

\section{Preoperative training}

Initially marmosets learned to respond to visual stimuli presented on the VDU to receive access to $5 \mathrm{sec}$ of banana milkshake, pumped at 0.5 $\mathrm{ml} / \mathrm{min}$ (Roberts et al., 1992). Subsequently, they began preoperative acquisition of touchscreen responding under a second-order schedule of responding with conditioned reinforcement.

Marmosets were tested once daily, in the afternoon. Each session lasted for $20 \mathrm{~min}$. Monkeys were presented with two identical blue squares, one on either side of the touch-sensitive VDU. One of the stimuli was designated the positive stimulus, responses to which led (1) to the presentation of the primary reinforcer ( $5 \mathrm{sec}$ of banana milkshake, pumped at $0.5 \mathrm{ml} / \mathrm{min}$ ) and (2) to the simultaneous presentation (for the duration of the primary reinforcer) of an auditory tone stimulus. This tone always accompanied (for the exact duration) the presentation of primary reinforcement and thus became a powerful conditioned stimulus and operated as the putative conditioned reinforcer. The other negative, visual stimulus acted as a control, with responses to it having no programmed consequence. A response to the positive visual stimulus resulted in the disappearance of both stimuli for $0.3 \mathrm{sec}$, whereas a response to the negative visual stimulus led to their disappearance for 1 sec. On those occasions when a response was followed by the presentation of the tone or of both the tone and primary reinforcer, the VDU also remained blank for their duration. Because some of the later second- 


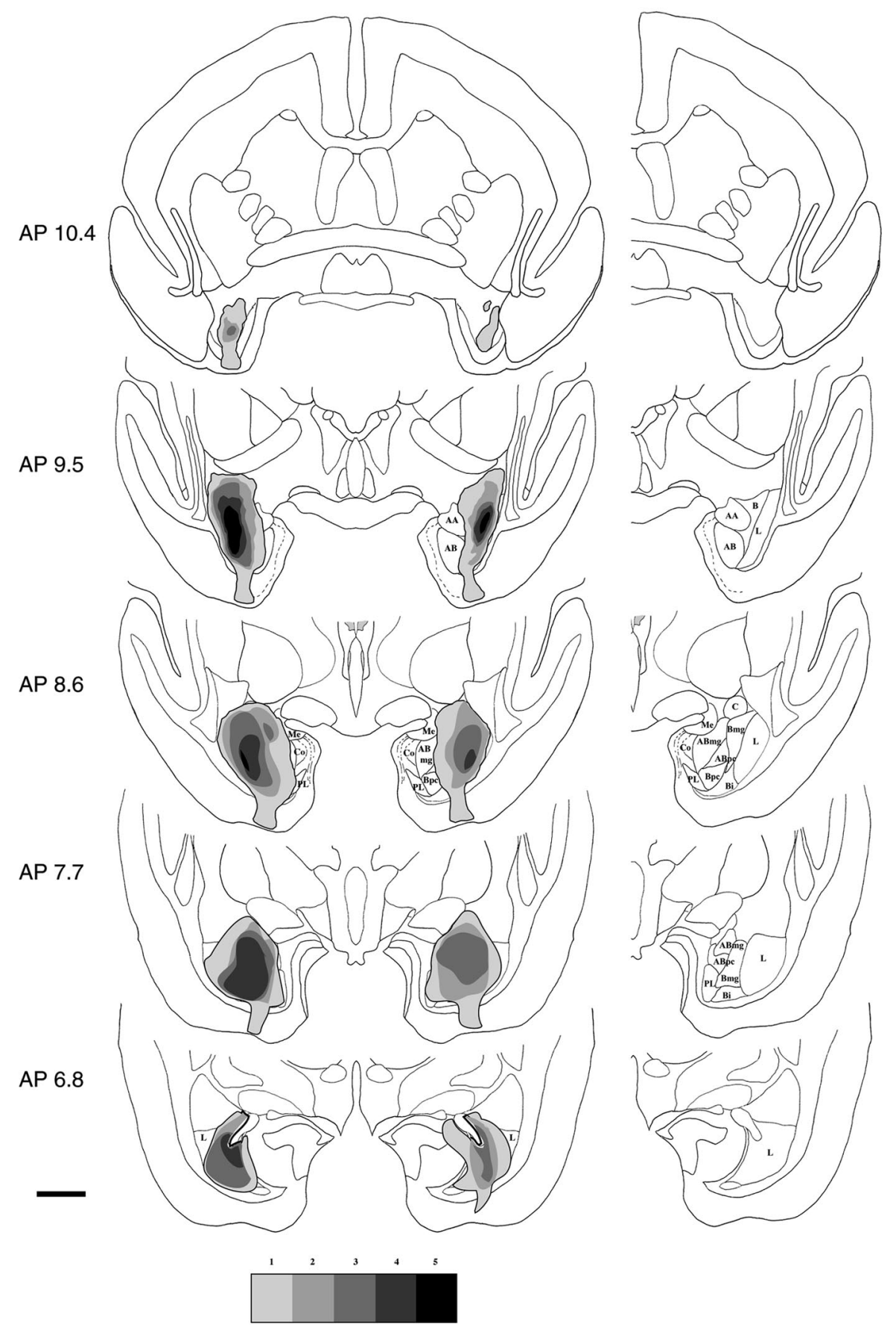

Figure 1. A schematic diagram of a series of coronal sections through the anterior temporal lobe (extended from $10.8-6.1 \mathrm{~mm}$ anterior to the interaural line in the anteroposterior plane) illustrating the extent of overlap of the amygdala lesions across the six monkeys. In this study, the excitotoxic lesions were targeted at the lateral and basal nuclei of the amygdala. [The location of the subnuclei can be seen in the single hemisphere to the right of the figure, abbreviated as lateral nucleus $(L)$, basal nucleus $(B)$, basal nucleus magnocellular subdivision $(B m g)$, basal nucleus parvocellular subdivision $(B p c)$, and basal intermediate nucleus (Bi).] The five levels of shading show, from lightest to darkest, the amount of tissue lesioned in at least one, two, three, four, and five marmosets, respectively. Overall, the figure provides a clear indication of both the overlap of damage and sparing of tissue in the lesioned monkeys. Because of the intrinsic variability in the location of the amygdala between marmosets, one discrete lesion was focused on the anterior and lateral amygdala (case 1) and another was focused more caudally and ventrally within the amygdala (case 6); these two did not show bilateral overlap in the extent of their lesions. Thus, only five levels of shading are presented in the figure. Refer to the histological analysis in Results for an additional description of these lesions by individual case. Scale bar, 400 $\mu \mathrm{m}$. $A A$, Anterior amygdala; $A B$, accessory basal nucleus; $A B m g$, magnocellular subdivision of the $\mathrm{AB} ; A B p c$, parvocellular subdivision of the $\mathrm{AB} ; C$, central nucleus; $C o$, cortical region of the amygdala; $M e$, medial nucleus; $P L$, paralaminar nucleus. order schedules took several minutes to complete, a contingency was included in the program such that if a marmoset was part-way through an individual schedule when the session time limit of 20 min was reached, these animals were given a maximum of 5 additional min to complete that schedule. Thus the session ended when either $5 \mathrm{~min}$ had elapsed or the marmoset had completed the schedule and been presented with the conditioned stimulus and primary reinforcer.

Marmosets determined their individual assignment of the sides of the screen for the positive and negative visual stimuli based on their first response to the blue stimuli during the initial session (i.e., the first response they made was reinforced with the tone and the primary reinforcer). The visual stimulus on the side to which they responded subsequently became the positive stimulus for the entire experiment. Once animals had made $>50$ responses in each of three consecutive sessions, the response requirement for the presentation of primary reinforcement was then increased every third session (i.e., a progressive second-order schedule of responding with conditioned reinforcement).
Animals were required to make two, three, and then five responses for a single presentation of primary reinforcement, with each response to the positive stimulus being accompanied by a brief $1 \mathrm{sec}$ presentation of the tone. These schedules can be described as FR2(FR1:S), FR3(FR1:S), and FR5(FR1:S), respectively, and will be described subsequently in terms of changes to the unit schedule $x$ and the brief stimulus presentation schedule $y$ in the equation $\mathrm{FR} x(\mathrm{FR} y: \mathrm{S})$.

At this final stage, it became clear that the magnitude of primary reinforcement (a $5 \mathrm{sec}$ presentation at $0.05 \mathrm{ml} / \mathrm{min}$ ) was insufficient ultimately to maintain protracted levels of responding; therefore, once marmosets had completed three sessions at FR5(FR1:S) the availability of the primary reinforcer was increased to a $10 \mathrm{sec}$ presentation of banana milkshake at $0.05 \mathrm{ml} / \mathrm{min}$ for all subsequent testing. Animals were maintained on this schedule for between six and ten sessions to enable a new level of stable responding to be acquired. Food and water restriction regimes ceased and animals then received surgery. 


\section{Postoperative training}

Second-order schedule. After 2 weeks of recovery from surgery, food and water restriction regimes were reintroduced and animals were retrained on the schedule FR5(FR1:S) until responding returned to presurgical levels. Indeed, all animals made equivalent or more responses within three postsurgical sessions, and thereafter the response requirements for the second-order schedule were increased every third session (as outlined below). If an animal failed to gain at least one primary reinforcer during each of three consecutive sessions, it was deemed to have failed at that level and received no additional testing. If an animal failed an individual session (i.e., failed to gain at least one primary reinforcer) it remained on that schedule until it either (1) successfully completed three consecutive sessions in which it gained at least one primary reinforcer in each session, resulting in progression to the next level, or (2) failed to gain a primary reinforcer across three consecutive sessions, resulting in removal from the schedule.

Initially, the $y$ schedule was increased to two, three, and then five responses [i.e., to FR5(FR5:S)]. From then on, the $y$ component was increased by two after every three sessions to a maximum final secondorder schedule of FR5(FR15:S). All subjects in the amygdala-lesioned group had dropped out by this stage, and this component of the experiment was then terminated.

The behavioral measures that were recorded included (1) mean number of responses to the positive stimulus across the three sessions of each schedule, (2) control stimulus responses, (3) other responses to areas outside the response boxes on the touchscreen, and (4) latency to collect the primary reinforcer.

CS omission. Additional testing was undertaken to determine the extent to which animals' responding on the second-order schedule was being maintained by the brief presentation of the conditioned stimulus, rather than by the progressively delayed presentation of primary reinforcement. The performance of marmosets was assessed on an omission test in which the conditioned stimulus is removed (Arroyo et al., 1998). Thus any reduction in responding on the schedule reflects the level of control by the conditioned stimulus in maintaining behavioral responding.

Two weeks after completion of the second-order schedule, animals were retrained starting at FR1(FR1:S). Animals followed the same progressive increase in the response demands of the second-order schedule until they reached a stable level of responding, with the added criteria that the level of primary reinforcement being received in a session was approximately equal across animals and groups (sham mean was 6.5 reinforcers per $20 \mathrm{~min}$ session; lesion mean was 6.9). Once animals demonstrated a stable level of responding for 3 consecutive days, the CS-omission procedure was introduced. An A-B-A design was used, such that animals were given two sessions on the second-order schedule (A), one session of CS omission (B), and then two additional sessions of the second-order schedule (A). During CS omission, all task parameters were identical to those of the previous second-order schedule except that the tone was omitted at the completion of the $y$ component of the schedule (i.e., the $1 \mathrm{sec}$ presentation) and also during the $10 \mathrm{sec}$ presentation of primary reinforcement.

Control schedule. To provide evidence that the effects of amygdala lesions were not attributable to disruptions in responding for primary reinforcement or to the ability of the marmosets to make accurate motoric responses to the screen, lesioned marmosets and their controls were tested on a progression of simple fixed-ratio schedules of primary reinforcement (i.e., a fixed number of responses to the touchscreen led to the presentation of the primary reinforcer). The visual stimulus that the animals were required to respond to was a blue "bow-tie"-shaped exemplar presented in the center of the screen. Auditory stimuli were not presented during any part of this control procedure, although all other parameters were identical to those of the second-order schedule. Animals began on an FR1 schedule which was then increased progressively so as to match the response requirement of the preceding second-order schedule. Hence, initial increments were FR1, FR3, FR5, etc. Subsequently, rather than the progression on the second-order schedule from, for example, FR5(FR5:S) to FR5(FR7:S), the comparable schedules were FR25 and FR35 (i.e., the number of responses required to obtain primary reinforcement were matched across the control and second-order schedules). Again, the schedule was increased every $3 \mathrm{~d}$ until animals no longer responded sufficiently to achieve primary reinforcement for 3 consecutive days.

\section{Statistical methods}

All behavioral data were analyzed using SPSS for Windows (version 9; SPSS, Inc., Chicago, IL). An overall ANOVA comparing responding across the second-order schedules was not possible, because the lesion and sham group sizes changed across the different schedules as individuals dropped out. Therefore, the data for the two groups at each level of the schedule [e.g., FR5(FR7:S)] were compared using independent $t$ tests adjusted for multiple comparisons (Bonferroni procedure) (Howell, 1999). In addition, the overall survival of animals from each group across the second-order schedule was compared using Fisher's exact (FE) statistic. The CS-omission data were analyzed using a repeated-measures ANOVA comparing responding before (mean of two pre-omission sessions), during, and after (mean of two post-omission sessions) the CSomission session. CS-omission data were analyzed using both the raw data and also a ratio measure to control for differences in baseline responding on different schedules. The ratio of responding was calculated as the mean number of responses during the CS-omission session divided by the sum of the mean number of responses during the pre-CS-omission session and the CS-omission session. Thus if the CS omission had no effect on the level of responding then the ratio score would be 0.5 . A score of $<0.5$ indicates a suppression in the CS-omission test. The post-CSomission ratio score was calculated as the mean number of responses during the post-CS-omission session divided by the sum of the mean number of responses during the pre-CS-omission session and the postCS-omission session. Both the pre-CS-omission and post-CS-omission scores were calculated as the mean from two baseline sessions to provide a stable value. The post-CS-omission score gives an indication as to whether responding returned to baseline levels when the CS was reintroduced after the CS-omission test (i.e., that the omission of the CS had not simply led to a global extinction of responding and that it could still control subsequent behavior).

\section{RESULTS}

\section{Histological assessment of lesions}

Lesions were analyzed using both cresyl fast violet-stained and NeuN-stained (Yakovlev et al., 1997) brain sections. Whereas cresyl violet identifies areas with necrotic neurons, gliosis, and tissue damage, NeuN provides a selective cell-body stain that enables a precise identification of cell-sparse lesioned areas. It should be noted that this study used the excitotoxin quinolinic acid to provide a selective means of lesioning the amygdala, sparing fibers of passage and overlying cortical tissue. Although there is some evidence that certain excitotoxins, namely NMDA, kainate, and ibotenic acid, can produce transient demyelination of fibers of passage (Brace et al., 1997), it has not been demonstrated that quinolinic acid produces such effects. Notwithstanding, the functional significance of such findings is as yet unknown.

A schematic representation of the extent of the amygdala lesions within the temporal lobe of all six lesioned monkeys is shown in Figure 1. This figure illustrates those regions of the amygdala that were consistently lesioned in all or nearly all marmosets and those regions that were only lesioned in a minority of marmosets. Given the interest in the contribution of extra-amygdala structures to deficits seen after amygdala ablation, it was our intention to produce discrete lesions within the amygdala while minimizing damage to extra-amygdala structures. Moreover, given the extensive literature regarding rats that has highlighted the importance of the basolateral area specifically in conditioned reinforcement, the focus of our lesion was intended to encompass primarily the basal and lateral nuclei (Fig. 1, $L, B, B m g, B p c, B i$ ) in the marmoset. As reflected in the darker shading of Figure 1, the focus of the lesions lies in the anterior lateral nucleus, with four of six marmosets displaying extensive cell loss throughout the anterior and middle parts of the lateral and basal nuclei. In addition, almost all monkeys showed some cell loss in the accessory 

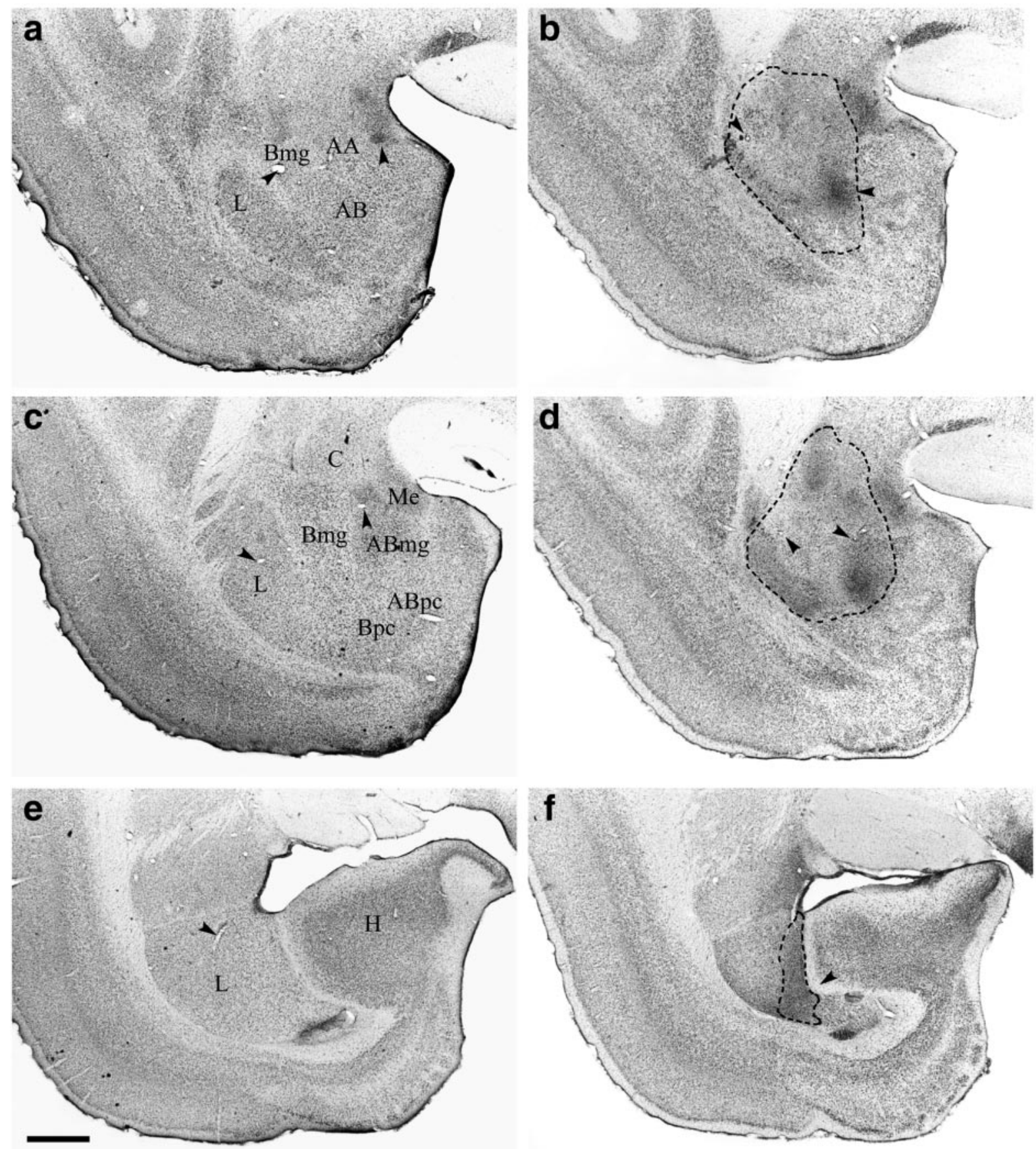

Figure 2. Photomicrographs of coronal sections through the amygdala of a representative amygdala lesion and a sham control. $a, c, e$ show low-power photomicrographs from a control monkey (approximately AP9.8, AP8.5, and AP7.1 anterior to the interaural line, respectively); $g, i, k$ show high-power photomicrographs of $a, c, e$, respectively; $b, d, f$ are low-power photomicrographs of the amygdala of a lesioned monkey taken at the same anteroposterior levels as the control sections $(a, c, e)$; and $h, j, l$ offer high-power magnification of the lesion depicted in $b, d, f$, respectively. Dotted lines show the extent of the lesion. Arrowheads highlight landmarks that should aid orientation and comparison of the high- and low-power pictures. In the ventromedial amygdala and parahippocampal gyrus, it can be seen that the tissue of the lesioned animal looks pale relative to the control sections. However, this area was not damaged by the lesion; the paleness is attributable to the variable nature of the cresyl stain, and the healthy nature of the individual cells in both animals can be seen in the high-power photomicrographs. Scale bars: $a-f, 200 \mu \mathrm{m} ; g-l, 100 \mu \mathrm{m}$. $A A$, Anterior amygdala; $A B$, accessory basal nucleus; $A B m g$, magnocellular subdivision of the AB; $A B p c$, parvocellular subdivision of the AB; Bmg, magnocellular subdivision of the basal nucleus; $B p c$, parvocellular subdivision of the basal nucleus; $C$, central nucleus; $H$, hippocampus; $L$, lateral nucleus; $M e$, medial nucleus. (Figure 2 continues.)

basal nucleus (five of six monkeys) and the central nucleus (four of six monkeys). No marmoset had damage in the cortical regions of the amygdala, and only two monkeys had partial damage to the medial nucleus. Only one marmoset with the most extensive amygdala lesion showed any extra-amygdala cortical damage (case 2, described below), bilaterally along the border between the parahippocampal gyrus and inferior temporal cortex, and restricted neuronal loss in the anterior medial hippocampus. Two animals also showed a small amount of cell loss dorsal to the amygdala in the region of the substantia innominata. 

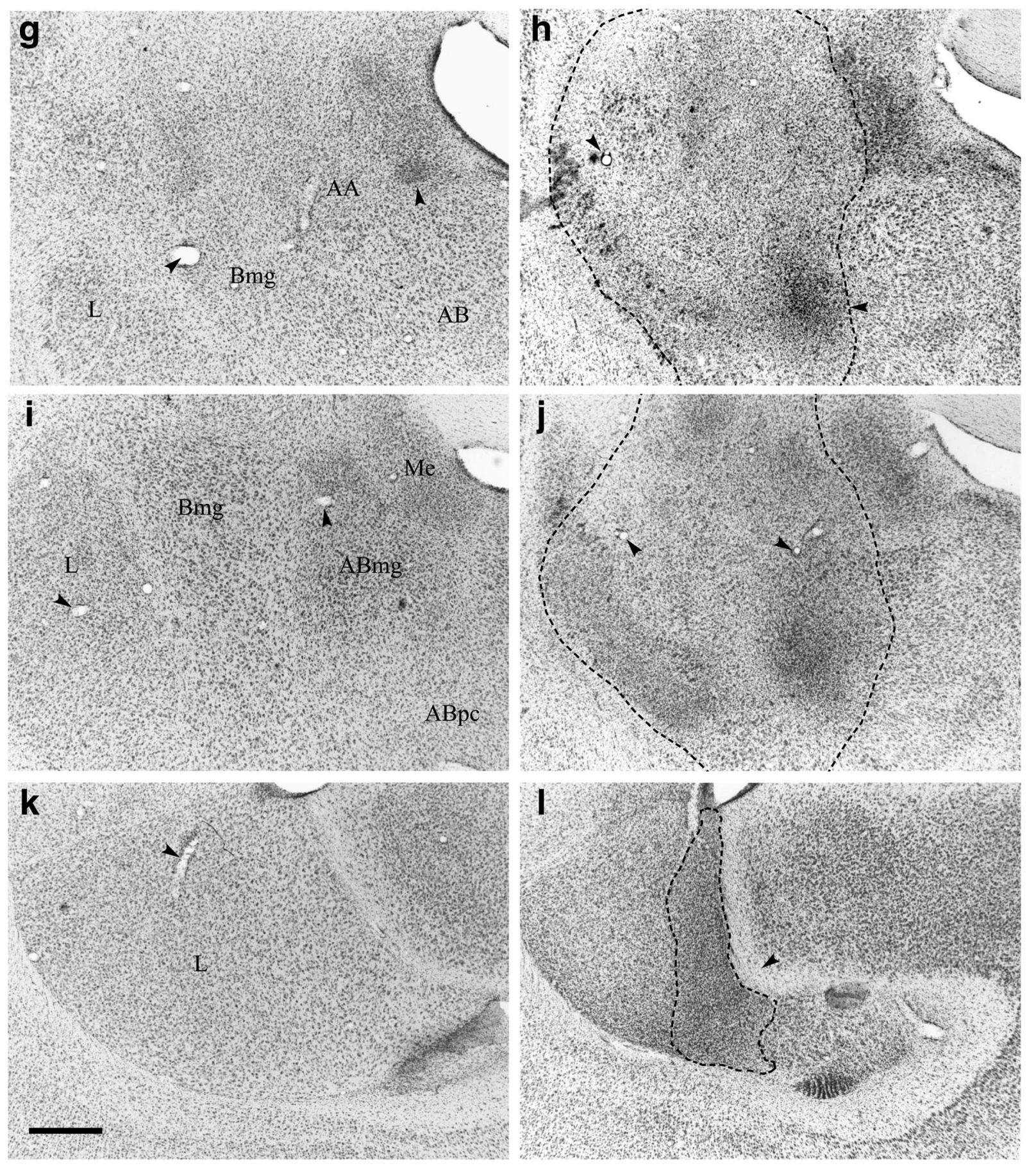

Figure 2 continued.

\section{Individual analyses}

\section{Case 1}

The lesion in this subject was bilateral and symmetrical, although relatively discrete and localized to the anterior lateral and basal nuclei. A large proportion of these two nuclei was lesioned, although the damage did not encompass the caudal region of these amygdala nuclei. However, caudally the lesion did include cell loss in the dorsal magnocellular subdivision of the accessory basal nucleus and encroached slightly on the medial nucleus. This lesion was the smallest of the six in this study.
Case 2

This lesion was the most extensive and included the entire rostrocaudal extent of the amygdala. The lateral nucleus was almost entirely destroyed bilaterally and both the magnocellular and parvocellular regions of the basal nucleus suffered cell loss, with only the most medial cells surviving. These intact cells were mainly within the caudal parvocellular region of the basal nucleus. The anterior region of the accessory basal nucleus was lesioned in its entirety in both hemispheres, with sparing of cells only in the more caudal and medial regions (from approximately AP8.0 in Fig. 1). The central nucleus suffered damage bilaterally, 
although more extensively on the left. Such damage was restricted to the lateral region of the central nucleus. The paralaminar nucleus showed a small amount of cell loss in the right hemisphere but remained intact in the left. At the level of the anterior hippocampus, damage was restricted to the dorsal region of the lateral nucleus on the left but the lesion spread ventrally throughout the lateral nucleus on the right.

There was a small amount of damage to the anterior temporal pole and also cell loss along the border between the parahippocampal gyrus and inferior temporal cortex along the entire anteroposterior extent of the amygdala. Finally, there was restricted neuronal loss in the anterior medial hippocampus (as can be seen in the caudal coronal section of Fig. 1).

\section{Case 3}

This animal's lesion was asymmetrical in that there was a large lesion in the left amygdala and a much more discrete lesion in the right. On the left, cell loss was observed in the anterior amygdala and almost the entire basal and accessory basal nuclei; a very small proportion of cells remained intact in the caudal, ventral, and medial aspect of the basal nucleus. Approximately half of the anterior lateral nucleus was lesioned combined with a complete lesion of the caudal region of the lateral nucleus. Only the very dorsal neurons in the central nucleus survived the lesion. Finally, the majority of cells in the paralaminar nucleus were lesioned. Thus this was the most extensive within-amygdala lesion, destroying almost the entire lateral, basal, accessory basal, and central nuclei, although only within the left hemisphere. There was also a small amount of cell loss dorsal to the amygdala around the level AP9-AP9.5. On the right, there was focal damage to the anterior magnocellular division of the basal nucleus and some damage to the medial and anterior aspect of the lateral nucleus. There was no other observable damage in this case.

\section{Case 4}

The infusion of excitotoxin in this animal produced a discrete and localized lesion of predominantly the lateral nucleus, approximately half of which was destroyed bilaterally and symmetrically. This cell loss was restricted to the anterior and medial aspect of the lateral nucleus. There was also a small amount of damage bilaterally, although greater on the left, in the magnocellular region of the basal nucleus. There were no other observable signs of damage to the amygdala in this animal.

\section{Case 5}

This animal's lesion was similar in extent to case 2, although with the important exception that, in this case, no extra-amygdala damage was observed in the temporal cortex. There was a small amount of unilateral (left) damage to the anterior amygdala. In addition, there was extensive bilateral damage to both the lateral and basal nuclei. This extended almost the full rostrocaudal extent of the amygdala, sparing only the ventral aspects of the lateral nucleus and the ventromedial aspect of the basal nucleus. There was cell loss in the lateral parvocellular region of the accessory basal nucleus, predominantly on the left, although almost the entire magnocellular region was spared bilaterally. The central nucleus was lesioned in its entirety on the left and almost completely on the right. The basal intermediate nucleus and paralaminar nucleus were spared bilaterally. In this animal, there was some bilateral cell loss dorsal to the amygdala around the region of the substantia innominata.

\section{Case 6}

The lesion in this case was similar to cases 2 and 5, although it was predominantly restricted to the caudal two-thirds of the amygdala. In this caudal region, the ventral lateral nucleus showed a significant amount of cell loss, as did almost the entire basal and accessory basal nuclei, with some sparing of the medial regions of these two nuclei. The central nucleus showed some damage unilaterally on the right and the medial nucleus showed a small amount of bilateral damage. The paralaminar nucleus was spared bilaterally.

In summary, four animals showed extensive lesions of the lateral, basal, and, to a lesser degree, accessory basal nuclei with some damage to other amygdala subnuclei including the central and medial nuclei. One of these four animals showed only a partial lesion on one side. The remaining two animals had discrete lesions centered on the lateral nucleus of the amygdala with a small amount of damage to the basal nucleus.

\section{Behavioral results}

\section{Second-order schedule: presurgical performance}

There were no differences in responding between the groups preoperatively. ANOVA of the mean responses (during the three sessions) on each schedule revealed a main effect of schedule $\left(F_{(2,20)}=8.58 ; p<0.01\right)$ but no effect of group $\left(F_{(1,10)}=0.01\right)$ and no interaction $\left(F_{(2,20)}=0.2\right)$. The schedule effect was attributable to an increase in responding as the schedule requirements increased.

\section{Second-order schedule: postsurgical performance}

After surgery, the most significant behavioral effect was that monkeys with amygdala lesions failed to reach later stages of the second-order schedule compared with their sham controls. Figure $3 A$ shows a survival plot for animals on the second-order schedule. Animals in the lesion group did not respond at sufficiently high enough rates to progress as far along the second-order schedule as sham controls. This was confirmed by Fisher's exact statistic (Siegal and Castellan, 1988), which revealed a significant difference in group survival at the schedules FR5(FR9:S) (FE, $p<0.03)$ and FR5(FR11:S) (FE, $p<0.01)$ with a trend toward a difference at FR5(FR7:S) (FE, $p=0.09)$. Thus lesioned monkeys were only capable of completing second-order schedules with low response requirements [i.e., FR5(FR5:S) and lower].

Amygdala-lesioned monkeys also tended to make fewer responses on the second-order schedules that they did complete compared with controls (Fig. 3B). Independent $t$ tests adjusted for multiple comparisons (Bonferroni procedure) were used to compare responding in the lesion and control groups on the first five postsurgical second-order schedules. The family-wise error rate was kept constant at $\alpha=0.05$ by adjusting the accepted significance level for individual $t$ tests to $\alpha / n=0.01$, where $n$ is the number of multiple comparisons. There was a significant reduction in responding on the schedule FR5(FR5:S) $(t=3.31 ; p<$ 0.01 ) in the lesion group (relative to controls) and a trend for reduced responding on the schedule FR5(FR3:S) $(t=2.01 ; p=$ $0.06)$. No significant differences were seen for the remaining three schedules [FR5(FR1:S), $t=1.33$; FR5(FR7:S), $t=-0.44$; and FR5(FR9:S), $t=-1.5]$.

\section{CS omission}

Omission of the CS resulted in a significant decline in responding of monkeys in the control group, as can be seen in Figure 4. In contrast, the omission of the CS did not affect the responding of 
A
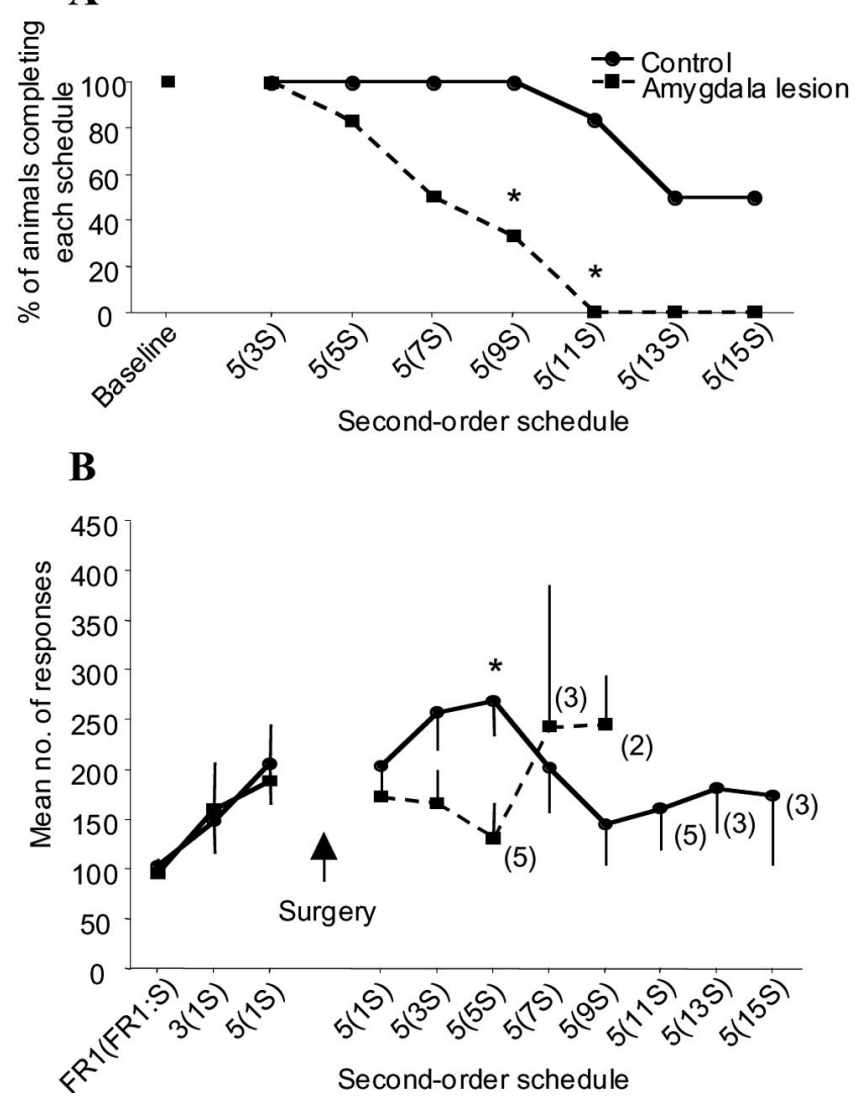

Figure 3. Effects of excitotoxic lesions of the amygdala on second-order responding with conditioned reinforcement; numbers along the abscissa refer to the type of second-order schedule; numbers in parentheses refer to the number of responses that have to be made to receive the CS. The numbers immediately outside the parentheses refer to the number of CS that have to be acquired before receiving primary reinforcement. For example, 5(7S) denotes a schedule in which seven responses leads to the presentation of the brief tone (CS) and primary reinforcement follows five such stimuli (i.e., a total of 35 responses). $A$, Survival plot of the number of animals successfully completing each stage of the second-order schedule. Animals with amygdala lesions dropped out significantly earlier than their sham controls. An asterisk denotes significance at $p<0.05$. B, Mean number of responses across each of the second-order schedules. There was a tendency for lesioned animals to make fewer responses relative to controls; this tendency reached significance at FR5(FR5:S) at $p<0.01$. Numbers in parentheses indicate the number of animals that remained in each group at each stage of the second-order schedule.

the amygdala-lesioned monkeys. Both the raw data for the CSomission test and a ratio of responding measure were analyzed separately using a repeated-measures ANOVA. Analysis of the raw response scores before (the mean of two pre-CS-omission sessions), during, and after (the mean of two post-CS-omission sessions) CS omission demonstrated that there was no overall difference in the level of responding of lesions and controls, as indicated by the lack of a main effect of lesion $\left(F_{(1,9)}=0.41\right)$ or of session $\left(F_{(2,18)}=0.62\right)$. However, a significant interaction of lesion $\times \operatorname{session}\left(F_{(2,18)}=3.62 ; p=0.048\right)$ revealed that responding in the control group was significantly reduced during the CS-omission session but not responding in the lesion group. An identical pattern of results was obtained after analysis of the ratio measure, with only the lesion $\times$ session interaction $\left(F_{(1,9)}=5.79\right.$; $p=0.039$ ) being significant.

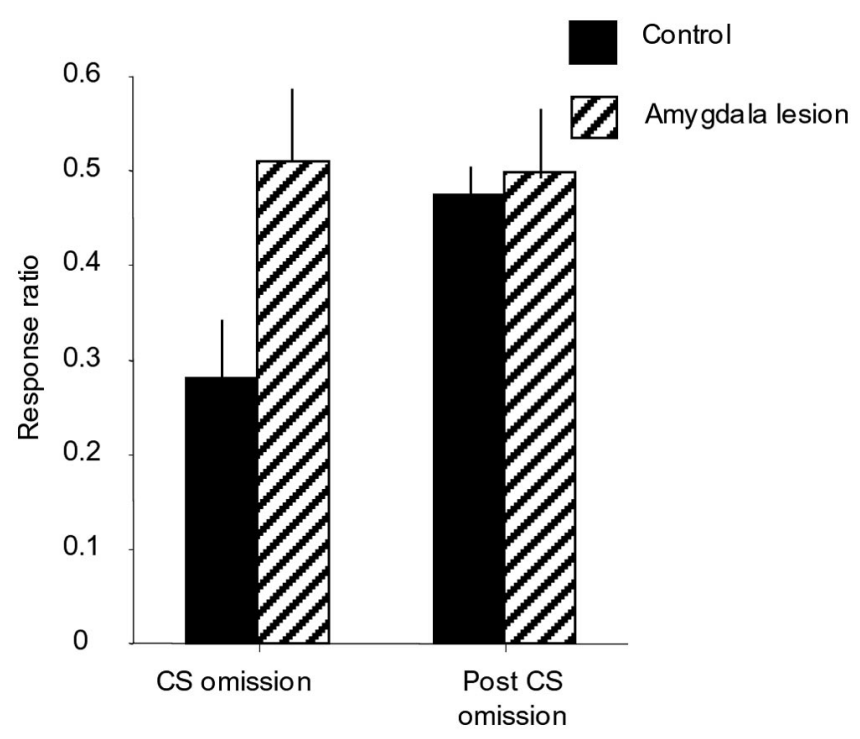

Figure 4. Comparison of the ratio of responding (see Statistical methods section in Material and Methods for equation) during the CS omission and after CS omission relative to responding before CS omission in control and amygdala-lesioned monkeys. A ratio of 0.5 indicates equivalent responding during CS omission or after CS omission compared with before CS omission. A ratio of $<0.5$ indicates suppression of responding. It can be seen that omission of the CS produced a reduction in responding in the control monkeys but not in the amygdala-lesioned animals [lesion $\times$ session interaction $\left.\left(F_{(1,9)}=5.79 ; p=0.039\right)\right]$. Responding in the control group returned to baseline levels when the CS was reintroduced.

\section{Control schedule}

To determine whether there was an impairment in responding for primary reinforcement after amygdala lesions that could have contributed to the deficit seen on the second-order schedule, all monkeys were tested on a progression of fixed-ratio schedules in the absence of any $\mathrm{CS}$. Figure $5 A, B$ shows that there were no marked differences between the lesioned and sham-operated monkeys with regard to their levels of responding or completion of the fixed-ratio schedules. The data were analyzed in a manner identical to that of the second-order task, with a Fisher's exact test assessing differences in survival (as defined previously) across the FR schedules and independent $t$ tests, adjusted for multiple comparisons, analyzing responding by the two groups on stages of the FR schedule.

These analyses showed that there were no significant effects of the amygdala lesion on this task. Analysis of survival (Fig. 5A) at each level of the schedule and compared across the two groups revealed no significant differences; the largest group differences were seen on the schedules FR35, FR45, and FR55 ( $p=0.41)$. Multiple $t$ tests comparing variables over the first seven fixed-ratio response schedules confirmed that there were no significant differences between the groups at any stage of the procedure; all $t$ values were $<2$ except for the schedule FR55 ( $t=2.1, p=0.28)$ (Fig. 5B).

\section{Second-order schedule: control measures}

Several other variables were also analyzed including (1) the number of responses to the control stimulus; (2) the mean latency to collect the primary reinforcer, once presented; (3) the mean trial length; and (4) the number of responses made to the touchscreen that did not fall within an appropriate stimulus area. None of these variables were affected by the amygdala lesion. Responding to the control stimulus was very low for all animals throughout 
A

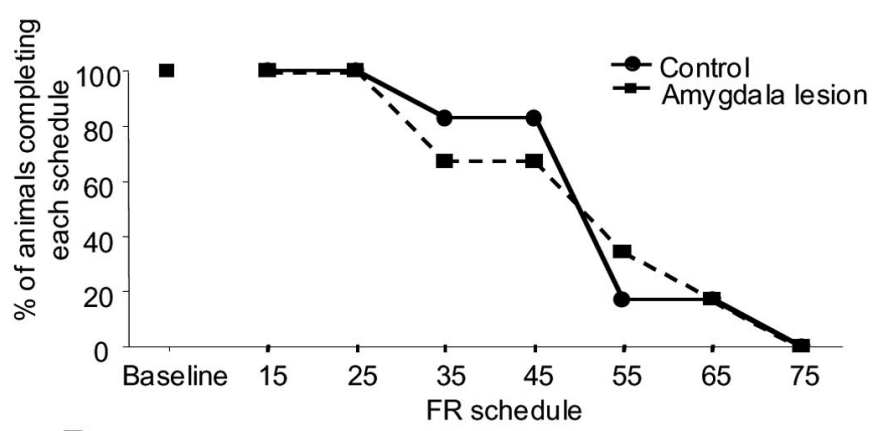

B

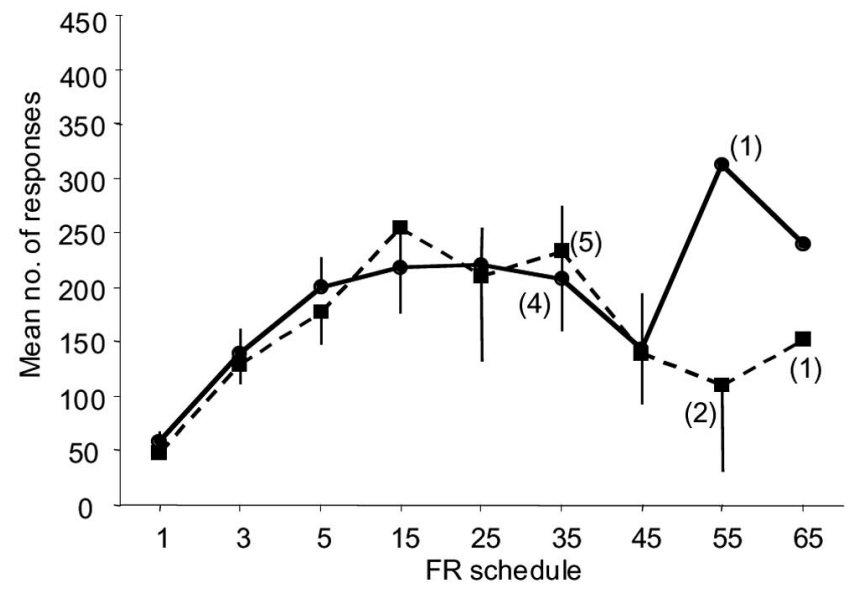

Figure 5. Effects of excitotoxic lesions of the amygdala on a progression of FR schedules for a primary reinforcer. Numbers along the abscissa refer to the type of FR schedule. $A$, Survival plot of the number of animals successfully completing each stage of the FR schedule. $B$, Mean number of responses across the FR. There were no group differences in survival or in the number of responses made across the FR schedules.

the experiment and was not significantly affected by the lesion, either before or after surgery (all $F$ and $t$ values $<2$ ). The latency to collect the primary reinforcer also did not differ between the groups either before or after surgery (all $F$ and $t$ values $<2$ ). In addition, trial length before or after surgery did not differ between groups. Presurgically, trial lengths increased across sessions, as the number of responses required to complete each schedule increased $\left(F_{(2,14)}=6.4 ; p<0.05\right)$, although there were no group differences (all $F$ values $<2$ ). Postsurgically, trial lengths increased in a relatively linear manner and showed no difference between groups at any subsequent level of the schedule (all $t$ values $<2$ ). Finally, the groups did not differ in the number of responses made outside of the appropriate stimulus areas (a measure of sensorimotor coordination). The mean level of inappropriate responding dropped progressively in both groups over testing, presumably because the animals became more proficient at making accurate stimulus-directed responses (all $F$ and $t$ values $<2$ ).

\section{Control schedule: control measures}

Again, there were no group differences in the latencies to collect the primary reinforcer or in the mean trial length across schedules between sham and lesion groups (all $t$ values $<2$ ).

\section{DISCUSSION}

Excitotoxic lesions of the amygdala in marmosets impaired performance on a second-order schedule of food reinforcement.
Specifically, as the schedule requirements increased, amygdalalesioned animals became progressively impaired at maintaining responding during protracted periods when such behavior was reinforced by the contingent presentation of the CS. That the responding of the sham-operated controls was under the control of the CS was indicated by the significant reduction in responding with its omission. In contrast, consistent with their poor performance under the second-order schedule, the responding of the amygdala-lesioned animals was insensitive to this manipulation. However, amygdala-lesioned monkeys were no different from sham controls in their ability to respond on a progression of fixed-ratio schedules for primary reinforcement. This latter test not only ruled out any gross motivational or general motor deficits produced by the amygdala lesion (Burns et al., 1993, 1999) but also demonstrated that responding governed by primary reinforcers, in contrast to that governed by conditioned reinforcers, was not significantly affected after the amygdala lesion. This pattern of results is consistent with a deficit in the ability of a CS, acting as a conditioned reinforcer, to support and control instrumental behavior. In addition, because the conditioned reinforcing properties of the stimulus were acquired before surgery in the present study, these findings demonstrate that the amygdala is critical for both the acquisition (Whitelaw et al., 1996) and performance (present study) of responding with conditioned reinforcement.

Although cross-species comparisons must be made with caution (D'Mello and Steckler, 1996; Roberts, 1996), the present results complement those in rats in which excitotoxic lesions, specifically of the basolateral area of the amygdala, disrupt responding for conditioned reinforcers on second-order schedules of sexual and drug reinforcement, similar to that used in the present study, while sparing responding for primary reinforcers (Everitt et al., 1989; Whitelaw et al., 1996). Indeed, such lesions have also been shown convincingly to disrupt acquisition of responding for a conditioned reinforcer that is performed in extinction (i.e., without primary reinforcement) (Cador et al., 1989; Burns et al., 1993), whereas lesions of the central nucleus of the amygdala were without effect (Robledo et al., 1996; for review, see Everitt et al., 2001). In contrast, the present results appear to be inconsistent with the recent demonstration of the lack of effect of excitotoxic amygdala lesions in monkeys on a second-order visual discrimination task (Malkova et al., 1997). However, on closer examination it can be seen that both studies consistently failed to show effects of amygdala lesions on low second-order schedules. Thus if primary reinforcement was available after only five or so responses, as was the case for the well trained monkeys in the study by Malkova et al. (1997) and for those monkeys at early postoperative stages of the present study, then no deficit was observed. Only when the response, and thereby the conditioned reinforcement requirements, increased further, as was the case in the present study, did amygdalalesioned monkeys fail to maintain responding.

This relative insensitivity of low second-order schedules to detect the effects of amygdala lesions on the conditioned reinforcement process may well be attributable to the additional, informational properties that conditioned stimuli possess that may support responding independently of any conditioned reinforcing process. Such additional properties may provide general information to the animal about the relationship of task events to one another as well as to the actions of the animal (e.g., the stimulus-stimulus association between the tone and food would endow the tone with predictive properties). In addition, a stimulus can bridge the temporal gap between a response and primary 
reinforcement and also provide feedback to an animal that its actions have had a causal impact on the environment (Williams, 1994). With respect to the study by Malkova et al. (1997), the extensive preoperative training that the monkeys received would have increased the likelihood that these additional properties of the auditory stimulus, other than that of conditioned reinforcement, guided discrimination learning. Although a monkey could not learn a visual discrimination based on primary reinforcement, nevertheless they always received primary reinforcement after each correctly performed discrimination. Thus, it is quite plausible that monkeys learned that responding to a stimulus that always resulted in the presentation of a particular tone led to the eventual availability of primary reinforcement, and thus it was the predictive rather than the affective properties of the tone that guided learning of new discriminations.

If the lack of effect seen after amygdala lesions in the study by Malkova et al. (1997) was indeed related to their procedure not actually taxing conditioned reinforcement mechanisms then this raises the question as to why Gaffan and Harrison (1987) observed a deficit on a second-order visual discrimination task after gross ablation of the amygdala. Certainly, Gaffan and Harrison (1987) used a less extensive training regime compared with that used by Malkova et al. (1997) and this may have reduced the likelihood of learning processes other than conditioned reinforcement controlling responding. However, it should be noted that in a later study the same authors showed that gross ablation of the amygdala did not affect second-order visual discrimination learning if a visual as opposed to an auditory stimulus acted as the CS (Gaffan et al., 1989). Thus, the deficit in the previous study with an auditory stimulus was more likely attributable to a disruption of other learning mechanisms (including cross-modal stimulusstimulus associations) caused by the extra-amygdala damage that follows ablation of the amygdala. Such damage includes destruction of fibers of passage, which effectively disconnects the temporal lobes from a variety of forebrain and brainstem structures, as well as incidental damage to the rhinal cortex (for a detailed discussion, see Malkova et al., 1997; Baxter et al., 1999).

The precise nature of the representation underlying conditioned reinforcement, and thus the role of the amygdala in this process, is not well understood. One hypothesis is that the conditioned stimulus elicits a general affective response. That conditioned stimuli in general may possess such properties has been demonstrated in a Pavlovian transreinforcer blocking experiment (Dickinson and Dearing, 1979) in which a stimulus that predicts one aversive event (i.e., shock) can block conditioning to a stimulus predicting another aversive event (i.e., the absence of an appetitive event). Because the nature of the aversive events is very different in the two cases, the only representation that the two stimuli have in common is their aversiveness, thus implicating a representation of "general affect" in controlling conditioning. However, whether conditioned reinforcement is based on such a representation has not been directly assessed. An alternative hypothesis is that the CS may act as a conditioned reinforcer by evoking a more specific affective representation of the nutritional or incentive value (Dickinson and Balleine, 1994) of the particular primary reinforcer with which it is associated. It is this latter process in which the amygdala has been specifically implicated (Malkova et al., 1997; Baxter et al., 2000), with the basolateral area appearing to be the critical locus (Hatfield et al., 1996; Schoenbaum et al., 1998) (for discussion of these issues, see Everitt et al., 2001).

In summary, this study contributes to the current literature regarding the critical role of the amygdala in both primates and nonprimates in the control of behavior by conditioned reinforcers. Although behavior can be influenced by a number of different properties of a conditioned stimulus (Mackintosh, 1974; Williams, 1994), one essential function of the amygdala and its associated circuitry, including the orbitofrontal cortex (Bechara et al., 1999; Baxter et al., 2000), appears to be to guide goal-directed actions based on the affective value of conditioned stimuli.

\section{REFERENCES}

Altman J, Everitt BJ, Glautier S, Markou A, Nutt D, Oretti R, Phillips GD, Robbins TW (1996) The biological, social, and clinical bases of drug-addiction: commentary and debate. Psychopharmacology 125:285-345.

Arroyo M, Markou A, Robbins TW, Everitt BJ (1998) Acquisition, maintenance, and reinstatement of intravenous cocaine selfadministration under a second-order schedule of reinforcement in rats: effects of conditioned cues and continuous access to cocaine. Psychopharmacology 140:331-344.

Baxter MG, Hadfield WS, Murray EA (1999) Rhinal cortex lesions produce mild deficits in visual discrimination learning for an auditory secondary reinforcer in rhesus monkeys. Behav Neurosci 113:243-252.

Baxter MG, Parker A, Lindner CC, Izquierdo AD, Murray EA (2000) Control of response selection by reinforcer value requires interaction of amygdala and orbital prefrontal cortex. J Neurosci 20:4311-4319.

Bechara A, Damasio H, Damasio AR, Lee GP (1999) Different contributions of the human amygdala and ventromedial prefrontal cortex to decision-making. J Neurosci 19:5473-5481.

Brace H, Latimer M, Winn P (1997) Neurotoxicity, blood-brain barrier breakdown, demyelination, and remyelination associated with NMDAinduced lesions of the rat lateral hypothalamus. Brain Res Bull 43:447-455.

Burns LH, Robbins TW, Everitt BJ (1993) Differential effects of excitotoxic lesions of the basolateral amygdala, ventral subiculum, and medial prefrontal cortex on responding with conditioned reinforcement and locomotor activity potentiated by intra-accumbens infusions of D-amphetamine. Behav Brain Res 55:167-183.

Burns LH, Everitt BJ, Robbins TW (1999) Effects of excitotoxic lesions of the basolateral amygdala on conditional discrimination learning with primary and conditioned reinforcement. Behav Brain Res 100:123-133.

Cador M, Robbins TW, Everitt BJ (1989) Involvement of the amygdala in stimulus reward associations: interaction with the ventral striatum. Neuroscience 30:77-86.

Damasio AR (1994) Descarte's error: emotion, reason and the human brain. London: Picador.

Dias R, Robbins TW, Roberts AC (1997) Dissociable forms of inhibitory control within prefrontal cortex with an analog of the Wisconsin Card Sort Test: restriction to novel situations and independence from "online" processing. J Neurosci 17:9285-9297.

Dickinson A, Balleine B (1994) Motivational control of goal-directed action. Anim Learn Behav 22:1-18.

Dickinson A, Dearing MF (1979) Appetitive-aversive interactions and inhibitory processes. In: Mechanisms of learning and motivation (Dickinson A, Boakes RA, eds), pp 203-231. Hillsdale, NJ: Erlbaum.

D’Mello GD, Steckler T (1996) Animal models in cognitive behavioural pharmacology: an overview. Brain Res Cogn Brain Res 3:345-352.

Everitt BJ (1990) Sexual motivation: a neural and behavioral analysis of the mechanisms underlying appetitive and copulatory responses of male rats. Neurosci Biobehav Rev 14:217-232.

Everitt BJ, Robbins TW (1992) Amygdala-ventral striatal interactions and reward-related processes. In: The amygdala (Aggleton JP, ed). New York: Wiley.

Everitt BJ, Cador M, Robbins TW (1989) Interactions between the amygdala and ventral striatum in stimulus reward associations: studies using a second-order schedule of sexual reinforcement. Neuroscience 30:63-75.

Everitt BJ, Cardinal RN, Hall J, Parkinson JA, Robbins TW (2001) Differential involvement of amygdala subsystems in appetitive conditioning and drug addiction. In: The amygdala: a functional analysis (Aggleton JP, ed), pp 353-390. Oxford: Oxford UP.

Gaffan D, Harrison S (1987) Amygdalectomy and disconnection in visual learning for auditory secondary reinforcement by monkeys. J Neurosci 7:2285-2292.

Gaffan D, Gaffan EA, Harrison S (1989) Visual-visual associative learning and reward-association learning in monkeys: the role of the amygdala. J Neurosci 9:558-564.

Gewirtz JC, Davis M (1997) Second-order fear conditioning prevented by blocking NMDA receptors in amygdala. Nature 388:471-474

Goldberg SR (1973) Comparable behavior maintained under fixed-ratio and second-order schedules of food presentation, cocaine injection, or 
D-amphetamine injection in the squirrel monkey. J Pharmacol Exp Ther 186:18-30.

Hatfield T, Han JS, Conley M, Gallagher M, Holland P (1996) Neurotoxic lesions of basolateral, but not central, amygdala interfere with Pavlovian second-order conditioning and reinforcer devaluation effects. J Neurosci 16:5256-5265.

Howell DC (1999) Fundamental statistics for the behavioral sciences. Belmont, CA: Duxbury Press.

Katz JL (1979) A comparison of responding maintained under secondorder schedules of intramuscular cocaine injection or food presentation in squirrel monkeys. J Exp Anal Behav 32:419-431.

Mackintosh NJ (1974) The psychology of animal learning. London: Academic.

Malkova L, Gaffan D, Murray EA (1997) Excitotoxic lesions of the amygdala fail to produce impairment in visual learning for auditory secondary reinforcement but interfere with reinforcer devaluation effects in rhesus monkeys. J Neurosci 17:6011-6020.

Meil WM, See RE (1997) Lesions of the basolateral amygdala abolish the ability of drug-associated cues to reinstate responding during withdrawal from self-administered cocaine. Behav Brain Res 87:139-148.

Roberts AC (1996) Comparison of cognitive function in human and non-human primates. Brain Res Cogn Brain Res 3:319-327.
Roberts AC, Robbins TW, Everitt BJ, Muir JL (1992) A specific form of cognitive rigidity following excitotoxic lesions of the basal forebrain in marmosets. Neuroscience 47:251-264.

Robledo P, Robbins TW, Everitt BJ (1996) Effects of excitotoxic lesions of the central amygdaloid nucleus on the potentiation of reward-related stimuli by intra-accumbens amphetamine. Behav Neurosci 110:981-990.

Schoenbaum G, Chiba AA, Gallagher M (1998) Orbitofrontal cortex and basolateral amygdala encode expected outcomes during learning. Nat Neurosci 1:155-159.

Siegal S, Castellan NJ (1988) Nonparametric statistics for the behavioral sciences. London: McGraw-Hill.

Whitelaw RB, Markou A, Robbins TW, Everitt BJ (1996) Excitotoxic lesions of the basolateral amygdala impair the acquisition of cocaineseeking behavior under a second-order schedule of reinforcement Psychopharmacology 127:213-224.

Williams BA (1994) Conditioned reinforcement: neglect or outmoded explanatory construct? Psychon Bull Rev 1:457-475.

Yakovlev AG, Knoblach SM, Fan L, Fox GB, Goodnight R, Faden AI (1997) Activation of CPP32-like caspases contributes to neuronal apoptosis and neurological dysfunction after traumatic brain injury. J Neurosci 17:7415-7424. 